\title{
On Lightlike Hypersurfaces of An Indefinite $f$-Kenmotsu Space Form
}

\author{
Yavuz Selim Balkan*
}

(Communicated by Johann T. DAVIDOV )

\begin{abstract}
In the present study, we consider a $f$-Kenmotsu space form $\bar{M}(c)$ and we investigate its lightlike hypersurfaces. We prove the non-existence of these type hypersurfaces of an $f$-Kenmotsu space form when $f$ is a constant function and it takes different values from $-c$ or $3 c$ and so we give a chracterization of lightlike hypersurfaces on a $f$-Kenmotsu space form. Finally, we obtain some related properties.

Keywords: Indefinite f-Kenmotsu space form; Lightlike hypersurface; Second fundamental form.

AMS Subject Classification (2010): Primary: 53D10 ; Secondary: 53C15; 53C25; 53C35.

${ }^{*}$ Corresponding author
\end{abstract}

\section{Introduction}

It is well-known that one has there different types of submanifolds in semi-Riemannian manifolds as spacelike, timelike and lightlike. These conditions are determined by the characteristic structure of the induced metric on the tangent space. When a semi-Riemannian manifold is given, we directly obtain a natural existence of lightlike subspaces, due to the degeneracy of the metric, there are fundamental differences between the study of lightlike submanifolds and classical theory of Riemannian and semi-Riemannian submanifolds ([4] and [8]). Moreover, this topis is quite new and it has a developable aspect. Many authors focus on this topic and they investigate lightlike hypersurfaces in different ambient spaces in ([1]-[3], [5], [6], [10]).

Motivated by the previous works, we investigate the characteristic properties of lightlike hypersurfaces of an indefinite $f$ Kenmotsu space form and we get some conditions on the non-existence of lightlike hypersurfaces of an $f$-Kenmotsu space form when $f$ is a constant function and it is not equal to $-c$ or $3 c$. Thus, we give a characterization of these hypersurfaces on $\bar{M}(c)$ and we obtain some related results. On the other hand, we compute Gauss and Codazzi equations of these type hypersurfaces.

\section{Preliminaries}

Let $\bar{M}$ be a $(2 n+1)$-dimensional differentiable manifold. We can say that it has a $(\varphi, \xi, \eta)$-structure if we have a $(1,1)$ tensor field $\varphi$, a vector field $\xi$ and a 1-form $\eta$ which satisfy

$$
\eta(\xi)=1 \quad \text { and } \quad \varphi^{2}=-I+\eta \otimes \xi
$$

where $I$ denotes the identity transformation. If the $\bar{M}$ endowed with a $(\varphi, \xi, \eta)$-structure admits a compatible semi-Riemannian metric $\bar{g}$ such that

$$
\bar{g}(\varphi x, \varphi y)=\bar{g}(x, y)-\varepsilon \eta(x) \eta(y) \quad \text { and } \quad \eta(x)=\varepsilon \bar{g}(x, \xi)
$$

Received : 11-May-2017, Accepted : 25-August-2017 
for all vector fields $x, y \in \chi(\bar{M})$, then we call it an indefinite almost contact metric manifold. Here,

$$
\bar{g}(\xi, \xi)=\varepsilon \quad \text { and } \quad \varepsilon=\left\{\begin{array}{cc}
1, & \text { if } \xi \text { is spacelike } \\
-1, & \text { if } \xi \text { is timelike. }
\end{array}\right.
$$

Furthermore an indefinite almost contact metric manifold $\bar{M}$ is called an indefinite $f$-Kenmotsu manifold if the following properties hold

$$
\left(\bar{\nabla}_{x} \varphi\right) y=f\{-\bar{g}(\varphi x, y) \xi+\eta(y) \varphi x\} \quad \text { and } \quad \bar{\nabla}_{x} \xi=f \varphi^{2} x
$$

where $f$ denotes a smooth function defined on $\bar{M}$. An indefinite $f$-Kenmotsu manifold is a natural extension of an $f$-Kenmotsu manifold defined by Olszak in [7].

Now, for an indefinite $f$-Kenmotsu manifold $\bar{M}$, if its Riemannian curvature tensor $\bar{R}$ satisfies

$$
\begin{aligned}
\bar{R}(x, y) z= & \frac{c-3 f}{4}\{\bar{g}(y, z) x-\bar{g}(x, z) y\} \\
& +\frac{c+f}{4}\{\bar{g}(\varphi y, z) \varphi x-\bar{g}(\varphi x, z) \varphi y \\
& -2 \bar{g}(\varphi x, y) \varphi z+\bar{g}(x, z) \eta(y) \xi \\
& -\bar{g}(y, z) \eta(x) \xi+\eta(x) \eta(z) y-\eta(y) \eta(z) x\}
\end{aligned}
$$

for all vector fields $x, y$ and $z$ on $\bar{M}$, then it is called an indefinite $f$-Kenmotsu space form and we denote it $\bar{M}(c)$. Moreover, if $f$ is a constant function which is equal to $\alpha$ then it is also called $\alpha$-Kenmotsu space form. Also, it is said that Kenmotsu space form is a 1-Kenmotsu space form.

Now, we recall some fundamental properties which we use in the next section from [9]. Let $(M, g, S(T M))$ be a lightlike hypersurface of semi-Riemannian manifold $(\bar{M}, \bar{g})$ and $\bar{\nabla}$ be the Levi-Civita connection on $\bar{M}$ with respect to $\bar{g}$, where $S(T M)$ denotes the screen distribution. Then we have

$$
\bar{\nabla}_{x} y=\nabla_{x} y+h(x, y)
$$

and

$$
\bar{\nabla}_{x} V=-A_{V} x+\nabla_{x}^{\perp} V
$$

for all vector fields $x, y \in \Gamma(T M)$ and $V \in \Gamma(\operatorname{tr}(T M))$, where $\operatorname{lt}(T M)$ is the lightlike transversal vector bundle of $M$. Moreover, it is said that $\nabla_{x} y, A_{V} x \in \Gamma(T M)$ and $h(x, y), \nabla_{x}^{\perp} V \in \Gamma(l \operatorname{tr}(T M))$ and also it can be easily seen that $\nabla$ is a torsion free linear connection on $M, h$ is a $\Gamma(\operatorname{ltr}(T M))$-valued symmetric $F(M)$-bilinear form on $\Gamma(T M), A_{V}$ is a $F(M)$-linear operator on $\Gamma(T M)$ and $\nabla^{\perp}$ is a linear connection on the vector bundle $l \operatorname{tr}(T M)$.

Let us suppose that $\{\widetilde{E}, \widetilde{N}\}$ is a pair of sections on $U \subset M$. Thus one can define a symmetric $F(U)$-bilinear form $\widetilde{B}$ and a 1-form $\rho$ on $U$ as follows:

$$
\widetilde{B}(x, y)=\bar{g}(h(x, y), \widetilde{E})
$$

and

$$
\rho(x)=\bar{g}\left(\nabla_{x}^{\perp} \tilde{N}, \widetilde{E}\right)
$$

for each $x, y \in \Gamma\left(\left.T M\right|_{U}\right)$. Hence, by using (2.5), (2.6) we locally get

$$
\bar{\nabla}_{x} y=\nabla_{x} y+\widetilde{B}(x, y) \widetilde{N}
$$

and

$$
\bar{\nabla}_{x} V=-A_{\widetilde{N}} x+\rho(x) \tilde{N}
$$

respectively, where $\widetilde{B}$ is called a local second fundamental form, $A_{\widetilde{N}}$ denotes a shape operator and $\nabla$ is the induced linear torsion free connection. Furthermore, (2.9) and (2.10) are called Gauss and Weingarten formulas of the lightlike hypersurface of $\bar{M}$, respectively.

Now, let $\widetilde{P}$ be the projection of $T M$ on $S(T M)$. Then the local Gauss and Weingarten formulas can be given by

$$
\nabla_{x} \widetilde{P} y=\nabla_{x}^{\circ} \widetilde{P} y+\widetilde{C}(x, \widetilde{P} y) \widetilde{E}
$$


and

$$
\nabla_{x} \widetilde{E}=-A_{\widetilde{E}}^{\circ} x-\rho(x) \widetilde{E}
$$

where $\nabla_{x}^{\circ} \widetilde{P} y, A_{\widetilde{E}}^{\circ} x \in S(T M)$ and $\widetilde{C}$ denotes a 1-form on $U$. Then we obtain

$$
g\left(A_{\widetilde{N}} x, \widetilde{P} y\right)=\widetilde{C}(x, \widetilde{P} y), \quad \bar{g}\left(A_{\widetilde{N}} x, \widetilde{N}\right)=0
$$

and

$$
g\left(A_{\widetilde{E}}^{\circ} x, \widetilde{P} y\right)=\widetilde{B}(x, \widetilde{P} y), \quad \bar{g}\left(A_{\widetilde{E}}^{\circ} x, \widetilde{N}\right)=0
$$

for all vector fields $x, y \in \Gamma(T M)$.

Let $\bar{R}$ and $R$ be curvature tensors with respect to the connections $\bar{\nabla}$ and $\nabla$, respectively. So we get a relation between $\bar{R}$ and $R$ as

$$
\begin{aligned}
\bar{R}(x, y) z= & R(x, y) z+A_{h(x, z)} y-A_{h(y, z)} x \\
& +\left(\nabla_{x} h\right)(y, z)-\left(\nabla_{y} h\right)(x, z) .
\end{aligned}
$$

Also, we state that the induced connection on $M$ satisfies

$$
\left(\nabla_{x} g\right)(y, z)=\widetilde{B}(x, y) \widetilde{\omega}(z)+\widetilde{B}(x, z) \widetilde{\omega}(y)
$$

for any $x, y, z \in \Gamma(T M)$, where $\widetilde{\omega}$ is a differential 1-form locally defined on $M$ as follow:

$$
\widetilde{\omega}(x)=\bar{g}(x, \tilde{N})
$$

for each $x \in \Gamma(T M)$.

\section{Lightlike Hypersurfaces of Indefinite $f$-Kenmotsu Space Forms}

Lemma 3.1. Let $M$ be a lightlike hypersurface of $\bar{M}(c)$. Then

(i) we compute the Gauss formulae of $M$ like that

$$
\begin{aligned}
R(x, y) z= & \frac{c-3 f}{4}\{\bar{g}(y, z) x-\bar{g}(x, z) y\} \\
& +\frac{c+f}{4}\{\bar{g}(\varphi y, z) \widetilde{\sigma} x-\bar{g}(\varphi x, z) \widetilde{\sigma} y \\
& -2 \bar{g}(\varphi x, y) \widetilde{\sigma} z+\bar{g}(x, z) \eta(y) \xi \\
& -\bar{g}(y, z) \eta(x) \xi+\eta(x) \eta(z) y \\
& -\eta(y) \eta(z) x\}-\widetilde{B}(x, z) A_{\widetilde{N}} y+\widetilde{B}(y, z) A_{\widetilde{N}} x .
\end{aligned}
$$

(ii) the Codazzi formulae of $M$ is given as

$$
\begin{aligned}
\left(\nabla_{y} h\right)(x, z)-\left(\nabla_{x} h\right)(y, z)= & \frac{c+f}{4}\{\bar{g}(\varphi y, z) \widetilde{v}(x) \\
& -\bar{g}(\varphi x, z) \widetilde{v}(y)-2 \bar{g}(\varphi x, y) \widetilde{v}(z)\} \widetilde{N}
\end{aligned}
$$

for any $x, y, z \in \Gamma(T M)$.

Proof. Let $M$ be a lightlike hypersurface of an indefinite $f$-Kenmotsu space form $\bar{M}(c)$. For any $x \in \Gamma(T M)$, we directly have

$$
\varphi x=\widetilde{\sigma} x+\widetilde{v}(x) \widetilde{N}
$$

where $\widetilde{v}(x)=g(x, V), V=-\varphi \widetilde{E}$ and $\widetilde{\sigma}$ is a tensor field of type $(1,1)$ defined on $M$. From (2.4) and (2.15), we deduce

$$
\begin{aligned}
\bar{R}(x, y) z= & \frac{c-3 f}{4}\{\bar{g}(y, z) x-\bar{g}(x, z) y\} \\
& +\frac{c+f}{4}\{\bar{g}(\varphi y, z) \varphi x-\bar{g}(\varphi x, z) \varphi y \\
& -2 \bar{g}(\varphi x, y) \varphi z+\bar{g}(x, z) \eta(y) \xi \\
& -\bar{g}(y, z) \eta(x) \xi+\eta(x) \eta(z) y-\eta(y) \eta(z) x\} \\
& -A_{h(x, z)} y+A_{h(y, z)} x-\left(\nabla_{x} h\right)(y, z)+(\nabla y h)(x, z) .
\end{aligned}
$$


By virtue of (3.3) and from (3.4), then we get (3.1) and (3.2) by considering the tangential and transversal vector bundle parts.

Lemma 3.2. For a lightlike hypersurface $M$ of an indefinite $f$-Kenmotsu space form $\bar{M}(c)$. We have the following

$$
\begin{aligned}
\bar{g}(R(x, \widetilde{E}) z, \widetilde{N})= & -\frac{c-3 f}{4} \bar{g}(x, z)-\frac{c+f}{4}\{\widetilde{v}(z) \widetilde{\omega}(\varphi x) \\
& +2 \widetilde{v}(x) \widetilde{\omega}(\varphi z)-\eta(x) \eta(z)\}
\end{aligned}
$$

Proof. It can be easily seen from Lemma 3.1.

Lemma 3.3. For a lightlike hypersurface $M$ of an indefinite $f$-Kenmotsu space form $\bar{M}(c)$. Then we have

$$
\widetilde{B}(y, U)=\widetilde{C}(y, V)
$$

for any $y \in \Gamma(T M)$, where $U=-\varphi \tilde{N}$.

Proof. By virtue of definition $\widetilde{B}$, we have

$$
\begin{aligned}
\widetilde{B}(y, \varphi \widetilde{N}) & =\bar{g}(h(y, \varphi \widetilde{N}), \widetilde{E})=\bar{g}\left(\bar{\nabla}_{y} \varphi \tilde{N}, \widetilde{E}\right) \\
& =-\bar{g}\left(\bar{\nabla}_{y} \widetilde{N}, \varphi \widetilde{E}\right)+\bar{g}\left(\left(\bar{\nabla}_{y} \varphi\right) \tilde{N}, \widetilde{E}\right) .
\end{aligned}
$$

From (2.3) and (2.13), it follows that

$$
\widetilde{B}(y, \varphi \widetilde{N})=-\bar{g}\left(\bar{\nabla}_{y} \widetilde{N}, \varphi \widetilde{E}\right)=g\left(A_{N} y, \varphi \widetilde{E}\right)=\widetilde{C}(y, \varphi \widetilde{E})
$$

which completes the proof.

Theorem 3.1. We can not find a lightlike hypersurface of an indefinite $f$-Kenmotsu space form $\bar{M}(c)$ with parallel second fundamental form where $f \neq-c$ for all values on $\bar{M}$.

Proof. We assume that $M$ is a lightlike hypersurface of $\bar{M}(c)$ which satisfies our hypothesis conditions. By setting $y=\widetilde{E}$ and $z=\varphi \widetilde{N}$ in (3.2), it yields that

$$
-\frac{3 c+3 f}{4}\{\widetilde{v}(x)-2 \bar{g}(x, \varphi \widetilde{E})\}=0
$$

and taking $x=\varphi \widetilde{N}$ in the last equation, we deduce that

$$
f=-c
$$

which is a contradiction. Thus we get desired result.

Theorem 3.2. We can not find a lightlike hypersurfaces of an indefinite $f$-Kenmotsu space form $\bar{M}(c)$ with parallel screen distribution where $f \neq 3 c$ for all values on $\bar{M}$.

Proof. We assume that $M$ is a lightlike hypersurface of $\bar{M}(c)$ which satisfies our hypothesis conditions. By using (2.4), then we derive

$$
\bar{g}(\bar{R}(\widetilde{E}, \varphi \widetilde{N}) \varphi \widetilde{E}, \widetilde{N})=\frac{3 c-f}{4} .
$$

Furthermore, we have

$$
\begin{aligned}
\bar{g}(\bar{R}(x, y) \widetilde{P} z, \widetilde{N})= & \bar{g}(R(x, y) \widetilde{P} z, \widetilde{N}) \\
= & \left(\nabla_{x} \widetilde{C}\right)(y, \widetilde{P} z)-\left(\nabla_{y} \widetilde{C}\right)(x, \widetilde{P} z) \\
& +\rho(y) \widetilde{C}(x, \widetilde{P} z)-\rho(x) \widetilde{C}(y, \widetilde{P} z)
\end{aligned}
$$


from [3]. By virtue of (3.6), we get

$$
\bar{g}(\bar{R}(\widetilde{E}, \varphi \widetilde{N}) \varphi \widetilde{E}, \widetilde{N})=0 .
$$

Now, by considering together (3.5) and (3.7) then it follows that

$$
f=3 c
$$

which is a contradiction and thus we complete the proof.

Lemma 3.4. Let us assume that $M$ is a lightlike hypersurface of an indefinite $f$-Kenmotsu manifold $\bar{M}$. If $V$ is a principle vector field, then we have

$$
\widetilde{B}(V, U)=\widetilde{C}(V, V)=0 .
$$

Proof. By using (2.3) and (2.9), it follows that

$$
\bar{\nabla}_{x} U=-\bar{\nabla}_{x} \varphi \tilde{N}=-\varphi \bar{\nabla}_{x} \tilde{N}-\left(\bar{\nabla}_{x} \varphi\right) \tilde{N}
$$

which means

$$
\nabla_{x} U+\widetilde{B}(x, U) \widetilde{N}=\varphi A_{\widetilde{N}} x-\rho(x) \varphi \widetilde{N}+\bar{g}(x, U) \xi .
$$

By virtue of (3.3) and from (3.8), then we derive that

$$
\nabla_{x} U+\widetilde{B}(x, U) \widetilde{N}=\widetilde{\sigma} A_{\widetilde{N}} x+\widetilde{v}\left(A_{\widetilde{N}} x\right) \widetilde{N}-\rho(x) \varphi \widetilde{N}+\bar{g}(x, U) \xi .
$$

Now by comparing the transversal vector bundle parts of both sides of the last equation, it yields that

$$
\widetilde{B}(x, U)=\widetilde{v}\left(A_{\widetilde{N}} x\right)=-g\left(A_{\widetilde{N}} x, \varphi \widetilde{E}\right)=\widetilde{C}(x, V)
$$

which gives us the assertion.

Lemma 3.5. We assume that $M$ is a lightlike hypersurface of an indefinite $f$-Kenmotsu space form $\bar{M}(c)$. We compute the Codazzi formulae like that

$$
\begin{aligned}
\left(\nabla_{x} A_{\widetilde{N}}\right) y-\left(\nabla_{y} A_{\widetilde{N}}\right) x= & \frac{c-3 f}{4}\{\widetilde{\omega}(y) x-\widetilde{\omega}(x) y\} \\
& +\frac{c+f}{4}\{\bar{g}(y, U) \varphi x-\bar{g}(x, U) \varphi y \\
& +2 \bar{g}(\varphi x, y) U+\widetilde{\omega}(x) \eta(y) \xi \\
& -\widetilde{\omega}(y) \eta(x) \xi\}+\rho(y) A_{\widetilde{N}} x-\rho(y) A_{\widetilde{N}} x .
\end{aligned}
$$

Proof. It can be easily seen by straightforward computations, thus we omit it.

Let us consider an orthonormal basis $\left\{e_{1}, \ldots, e_{n-2}, \ldots, e_{2 n-4}, \xi, \widetilde{E}, \varphi \widetilde{E}, \varphi \widetilde{N}\right\}$ of $\Gamma(T M)$ such that

$$
\varphi e_{i}=e_{n-2+i}, \quad \varphi e_{n-2+i}=-e_{i} \quad \text { and } \quad \varphi \xi=0
$$

for each $i=1, \ldots, m-2$.

Lemma 3.6. Let $M$ be a lightlike hypersurface of an indefinite $f$-Kenmotsu manifold $\bar{M}$. Then

$$
\begin{aligned}
A_{\widetilde{N}} U= & \sum_{i=1}^{2 n-4} \frac{\widetilde{C}\left(U, e_{i}\right)}{\varepsilon_{i}} e_{i}+\widetilde{C}(U, \xi) \xi \\
& +\widetilde{C}(U, U) V+\widetilde{C}(U, V) U
\end{aligned}
$$

and

$$
A_{\widetilde{N}} \widetilde{E}=\sum_{i=1}^{2 n-4} \frac{\widetilde{C}\left(\widetilde{E}, e_{i}\right)}{\varepsilon_{i}} e_{i}+\widetilde{C}(\widetilde{E}, \xi) \xi+\widetilde{C}(\widetilde{E}, U) V
$$

where $\left\{\varepsilon_{i}\right\}$ denotes the signature of the basis $\left\{e_{i}\right\}$. 
Proof. By virtue of assumption, we can write

$$
A_{\widetilde{N}} U=\sum_{i=1}^{2 n-4} \lambda_{i} e_{i}+\gamma \xi+\alpha_{1} \widetilde{E}+\alpha_{2} \varphi \widetilde{E}+\alpha_{3} \varphi \widetilde{N} .
$$

By taking into account of (2.13), then we deduce that $\lambda_{i}=\frac{\widetilde{C}\left(U, e_{i}\right)}{\varepsilon_{i}}, \gamma=\widetilde{C}(U, \xi), \alpha_{1}=0, \alpha_{2}=-\widetilde{C}(U, U)$ and $\alpha_{3}=-\widetilde{C}(U, V)$. Thus it yields (3.9). In a similar way, we obtain (3.10).

Theorem 3.3. There are no lightlike hypersurfaces of an indefinite $f$-Kenmotsu manifold $\bar{M}$ with $f \neq 3 c$ satisfying

$$
g\left(\left(\nabla_{\widetilde{E}} A_{\widetilde{N}}\right) U, V\right)=g\left(\left(\nabla_{U} A_{\widetilde{N}}\right) \widetilde{E}, V\right)
$$

and

$$
\widetilde{B}(U, U)=0 .
$$

Proof. Putting $y=U$ and $x=\widetilde{E}$ in Lemma 3.5, it follows that

$$
\left(\nabla_{\widetilde{E}} A_{\widetilde{N}}\right) U-\left(\nabla_{U} A_{\widetilde{N}}\right) \widetilde{E}=-\frac{3 c-f}{4} U+\rho(U) A_{\widetilde{N}} \widetilde{E}-\rho(\widetilde{E}) A_{\widetilde{N}} U .
$$

By using (3.9) and (3.10), then we arrive at

$$
\begin{aligned}
\left(\nabla_{\widetilde{E}} A_{\widetilde{N}}\right) U-\left(\nabla_{U} A_{\widetilde{N}}\right) \widetilde{E}= & -\frac{3 c-f}{4} U+\rho(U)\left\{\frac{\widetilde{C}\left(\widetilde{E}, e_{i}\right)}{\varepsilon_{i}} e_{i}\right. \\
& +\widetilde{C}(\widetilde{E}, \xi) \xi+\widetilde{C}(\widetilde{E}, U) V\} \\
& -\rho(\widetilde{E})\left\{\frac{\widetilde{C}\left(U, e_{i}\right)}{\varepsilon_{i}} e_{i}+\widetilde{C}(U, \xi) \xi\right. \\
& +\widetilde{C}(U, U) V+\widetilde{C}(U, V) U\} .
\end{aligned}
$$

By taking into account of Lemma 3.4, then we derive

$$
g\left(\left(\nabla_{\widetilde{E}} A_{\widetilde{N}}\right) U-\left(\nabla_{U} A_{\widetilde{N}}\right) \widetilde{E}, V\right)=-\frac{3 c-f}{4} U-\rho(\widetilde{E}) \widetilde{B}(U, U)
$$

which implies the desired result.

Acknowledgement. The author is supported by the Düzce University Scientific Research Projects [Project number 2017.05.04.528]

\section{References}

[1] Aktan, N., On non-existence of lightlike hypersurfaces of indefinite Sasakian space form. Inter. J. Math. Statistic. 3 (A08) (2008), 12-21.

[2] Aktan, N., On non-existence of lightlike hypersurfaces of indefinite Kenmotsu space form. Turk. J. Math. 32 (2008), 127-139.

[3] Bejancu, A., Null hypersurfaces in semi-Euclidean space. Saitama Math. J. 14 (1996), 25-40.

[4] Duggal, K. L. and Bejancu, A., Lightlike submanifolds of semi-Riemannian manifolds and its applications, Kluwer Dortrecth, 1996.

[5] Güneş R., Şahin, B. and Kılıç, E., On Lightlike hypersurfaces of a semi-Riemannian space form. Turk. J. Math., 27 (2003), 283-297. 
[6] Kang, T. H., Jung, S. D., Kim, B. H., Pak, H. K. and Pak, J. S., Lightlike hypersurfaces of indefinite Sasakian manifolds. Indian J. Pure App. Math., 34 (2003), 1369-1380.

[7] Olszak, Z., Locally conformal almost cosymplectic manifolds. Coll. Math. LVII (1989), no. 1, 73-87.

[8] O’Neill, B., Semi-Riemannian geometry, AcademicPress, 1983.

[9] Duggal, K. L. and Sahin, B., Differential geometry of lightlike submanifolds, Birkhäuser, 2010.

[10] Sahin, B. and Güneş, R., Non-existence of real lightlike hypersurfaces of an indefinite complex space form. Balkan J. Geom. Appl. 5 (2000), no. 2, 139-148.

\section{Affiliations}

YAVUZ SELIM BALKAN

ADDRESS: Düzce University, Faculty of Arts and Sciences, Department of Mathematics, 81620, Düzce-TURKEY. E-MAIL: y.selimbalkan@gmail.com 